\title{
Stability and Hopf Bifurcation Analysis of Delayed Rosenzweig-MacArthur Model with Prey Immigration
}

\author{
Gang Zhu*, Chunyan He \\ School of Education Science, Harbin University, Harbin, P. R. China
}

Email address:

zhuganghit@163.com (Gang Zhu)

\section{To cite this article:}

Gang Zhu, Chunyan He. Stability and Hopf Bifurcation Analysis of Delayed Rosenzweig-MacArthur Model with Prey Immigration American Journal of Applied Mathematics. Vol. 7, No. 6, 2019, pp. 164-176. doi: 10.11648/j.ajam.20190706.14

Received: October 25, 2019; Accepted: November 18, 2019; Published: December 30, 2019

\begin{abstract}
A delayed reaction Cdiffusion Rosenzweig-MacArthur model with a constant rate of prey immigration is considered. We derive the characteristic equation through partial differential equation theory, and by analyzing the distribution of the roots of the characteristic equation, the local stability of the positive equilibria is studied, and we get the conditions to determine the stability of the positive equilibria. Furthermore we find that Hopf bifurcation occurs near the positive equilibrium when the time delay passes some critical values, and we get the conditions under which the Hopf bifurcation occurs and so periodic solutions appear near the positive equilibria. By using the center manifold theory and normal form method, we derive an explicit algorithm for determining the direction of the Hopf bifurcation and the stability of the bifurcating periodic solutions. Furthermore, some numerical simulations are carried out to illustrate the analytic results of our study.
\end{abstract}

Keywords: Delay, Stability, Bifurcation, Center Manifold, Normal Form

\section{Introduction}

Predator-prey systems (or consumer-resource systems) are basic differential equation models for describing the interactions between two species with a pair of positivenegative feedbacks. Furthermore, many ecological concepts such as diffusion, functional responses and time delays have been taken into consideration to gain more accurate description and better understanding [1-6]. A typical RosenzweigMacArthur model was put forward first in [7]. In this model the prey has a logistic growth and the predator has a Holling
II functional response. In [8-12] the global stability are discussed. There are also many researches on the limit cycle of R-M model [13-16]. In [17], Malay Banerjeethe studied the existence of Turing patterns in this model and in the effect of the non-local interaction on the periodic travelling wave and spatio-temporal chaotic patterns. In [18], a fractional order Rosenzweig-MacArthur (R-M) model incorporating a prey refuge is constructed and analyzed in detail. Sugie et al discussed the existence and uniqueness of limit cycles in predator-prey systems with a constant immigration in [19].

In this paper, we study the reaction-diffusion system, Rosenzweig-MacArthur model with a constant immigration.

$$
\begin{gathered}
\frac{\partial u(t, x)}{\partial t}=D_{1} \Delta u(t, x)+r u(t, x)\left[1-\frac{u(t, x)}{k}\right]-\frac{u(t, x) v(t, x)}{a+u(t, x)}+b \frac{\partial v(t, x)}{\partial t}=D_{2} \Delta v(t, x)-d v(t, x)+ \\
\frac{\mu u(t-\tau, x) v(t-\tau, x)}{a+u(t-\tau, x)}, \quad x \in \Omega, \quad t>0 \frac{\partial u(t, x)}{\partial \nu}=\frac{\partial u(t, x)}{\partial \nu}=0 \\
x \in \partial \Omega, \quad t \geq 0 u(t, x)=\phi(t, x) \geq 0, \quad v(t, x)=\psi(t, x) \geq 0, \quad x \in \bar{\Omega}, \quad \theta \in[-\tau, 0]
\end{gathered}
$$


Where $\Omega=(0, \pi)$, the homogeneous Neumann boundary condition means the system is a closed one. $u(t, x), v(t, x)$ stand for the densities of the prey and predator at time $t>0$ and a spatial position $x \in \Omega, D_{1}, D_{2}>0$ are the diffusion coefficients of the species. $a, b, d, k, r, \mu$ are all positive constants, and the meaning of them are the same as those in [19], and $\tau \geq 0$ is the constant delay due to the gestation of the predator.

It is easy to see that system (1) always has a boundary equilibrium

$$
E_{0}\left(u_{0}, 0\right)=\left(\frac{k}{2}+\frac{\sqrt{k^{2} r^{2}+4 b k r}}{2 r}, 0\right)
$$

When $\mu>d$, we let

$$
R_{0}=\frac{b k(\mu-d)^{2}+a d k r(\mu-d)}{a^{2} d^{2} r}
$$

then we can get that when $R_{0} \leq 1$ system (1) has no positive equilibrium and $E_{0}\left(x_{0}, 0\right)$ is the unique equilibrium of (1) ; and when $R_{0}>1$ system (1.1) has a positive equilibrium $E_{*}\left(u_{*}, v_{*}\right)$ besides $E_{0}\left(u_{0}, 0\right)$,

$$
E_{*}\left(u_{*}, v_{*}\right)=\left(\frac{a d}{\mu-d}, \frac{\mu}{d}\left[r u_{*}\left(1-\frac{u_{*}}{k}\right)+b\right]\right)
$$

$$
\begin{array}{r}
\frac{\partial u(t, x)}{\partial t}=D_{1} \triangle u(t, x)+\alpha_{1} u(t, x)-\alpha_{2} v(t, x)+f_{1}(u, v) \frac{\partial v(t, x)}{\partial t}=D_{2} \Delta v(t, x)- \\
d v(t, x)+\beta_{1} u(t-\tau, x)+d v(t-\tau, x)+f_{2}(u, v) \frac{\partial u(t, x)}{\partial \nu}=\frac{\partial u(t, x)}{\partial \nu}=0, \\
x \in \partial \Omega, \quad t \geq 0 u(t, x)=\phi(t, x)-u_{*}, \quad v(t, x)=\psi(t, x)-v_{*}, \quad x \in \bar{\Omega}, \quad \theta \in[-\tau, 0]
\end{array}
$$

where

$$
\begin{gathered}
\alpha_{1}=r-\frac{2 r u_{*}}{k}-\frac{a v_{*}}{\left(a+u_{*}\right)^{2}}, \quad \alpha_{2}=\frac{u_{*}}{a+u_{*}}, \quad \beta_{1}=\frac{a \mu v_{*}}{\left(a+u_{*}\right)^{2}} \\
f_{1}(u, v)=-\frac{r u(t, x)^{2}}{k}+\frac{a v_{*} u^{2}(t, x)-a\left(a+u_{*}\right) u(t, x) v(t, x)}{\left(a+u_{*}\right)^{2}\left(a+u_{*}+u(t, x)\right)} \\
f_{2}(u, v)=a \mu \frac{\left(a+u_{*}\right) u(t-\tau, x) v(t-\tau, x)-v_{*} u^{2}(t-\tau, x)}{\left(a+u_{*}\right)^{2}\left(a+u_{*}+u(t-\tau, x)\right)}
\end{gathered}
$$

Denotinig $X=C\left([0, \pi], \mathbb{R}^{2}\right), C_{\tau}=C([-\tau, 0], X)$

$$
u_{1}=u(t, \cdot), u_{2}=v(t, \cdot), \quad U=\left(u_{1}, u_{2}\right)^{T}
$$

then we can write (2) as an abstract ordinary differential equation in the phase space $C_{\tau}$

$$
\dot{U}(t)=D \triangle U(t)+L\left(U_{t}\right)+F\left(U_{t}\right)
$$

where

$$
\begin{aligned}
\operatorname{dom}(D \triangle) & =\left\{(u, v)^{T} \mid u, V \in C^{2}([0, \pi], \mathbb{R}), \partial_{\nu} u=\partial_{\nu} v=0, \pi\right\} \\
L(\phi) & =\left(\begin{array}{cc}
\alpha_{1} & -\alpha_{2} \\
0 & 0
\end{array}\right)\left(\begin{array}{c}
\phi_{1}(0) \\
\phi_{2}(0)
\end{array}\right)+\left(\begin{array}{cc}
0 & 0 \\
\beta_{1} & d
\end{array}\right)\left(\begin{array}{c}
\phi_{1}(-\tau) \\
\phi_{2}(-\tau)
\end{array}\right), \quad D=\left(\begin{array}{cc}
D_{1} & 0 \\
0 & D_{2}
\end{array}\right) \\
F(\phi) & =\left(\begin{array}{c}
-\frac{r \phi_{1}^{2}(0)}{k}+\frac{a v_{*} \phi_{1}^{2}(0)-a\left(a+u_{*}\right) \phi_{1}(0) \phi_{2}(0)}{\left(a+u_{*}\right)^{2}\left(a+u_{*}+\phi_{1}(0)\right)} \\
a \mu \frac{\left(a+u_{*}\right) \phi_{1}(-\tau) \phi_{2}(-\tau)-v_{*} \phi_{1}^{2}(-\tau)}{\left(a+u_{*}\right)^{2}\left(a+u_{*}+\phi_{1}(-\tau)\right)}
\end{array}\right) .
\end{aligned}
$$


Equation (4) has the linear part

$$
\dot{U}(t)=D \triangle U(t)+L\left(U_{t}\right)
$$

and the characteristic equation

$$
\lambda y-D \triangle y-L\left(\mathrm{e}^{\lambda \cdot} y\right)=0, \quad y \in \operatorname{dom}(D \triangle) \backslash\{0\}
$$

Letting

$$
\phi=\sum_{n=0}^{\infty}\left(\begin{array}{l}
a_{n} \\
b_{n}
\end{array}\right) \cos (n \pi x)
$$

be the characteristic function corresponding to the eigenvalues $\lambda$, then the characteristic equation has the following form

$$
\triangle_{n}(\lambda, \tau)=\lambda^{2}+A_{n} \lambda+B_{n}+\left(C_{n}-d \lambda\right) \mathrm{e}^{\lambda \tau}=0
$$

where

$$
\begin{aligned}
& A_{n}=\left(D_{1}+D_{2}\right) n^{2}+d-\alpha_{1}, \\
& B_{n}=D_{1} D_{2} n^{4}+\left(D_{1} d-D_{2} \alpha_{1}\right) n^{2}-d \alpha_{1}, \\
& C_{n}=-D_{1} d n^{2}+d \alpha_{1}+\alpha_{2} \beta_{1} .
\end{aligned}
$$

Assuming condition $(A)$ as follow:

$$
\text { (A) } \alpha_{1}<0 \Leftrightarrow r+\frac{a d r}{k \mu}<\frac{2 a d r}{k(\mu-d)}+\frac{r(\mu-d)}{\mu}+\frac{b(\mu-d)^{2}}{a d \mu},
$$

then we have Lemma 2.1. If $(A)$ holds, then $\lambda=0$ is not the root of Eq. (5) for any $n \in \mathbb{N}_{0}$.

Proof. According to (5) we know that

$$
\triangle_{n}(0, \tau)=B_{n}+C_{n}=D_{2} n^{2}\left(D_{1} n^{2}-\alpha_{1}\right)+\alpha_{2} \beta_{1}
$$

Condition $(A)$ holds means that $\alpha_{1}<0$, and from (3) we can get $\alpha_{2}>0, \beta_{1}>0$, then

$$
\triangle_{n}(0, \tau)>0, \forall n \in \mathbb{N}_{0}
$$

Lemma 2.2. If $(A)$ holds, when $\tau=0$, all roots of Eq. (5) have negative real parts, for any $n \in \mathbb{N}_{0}$, furthermore, $E_{*}$ is locally asymptotically stable.

Proof. When $\tau=0$, Eq. (5) becomes

$$
\triangle_{n}(\lambda, 0)=\lambda^{2}+\left(A_{n}-d\right) \lambda+B_{n}+C_{n}=0 .
$$

Suppose that $\lambda_{1 n}, \lambda_{2 n}$ be roots of the above equation, then we have

$$
\begin{array}{r}
\lambda_{1 n}+\lambda_{2 n}=d-A_{n}=\alpha_{1}-\left(D_{1}+D_{2}\right)<0 \\
\lambda_{1 n} \lambda_{2 n}=B_{n}+C_{n}=D_{1} D_{2} n^{4}-D_{2} \alpha_{1} n^{2}+\alpha_{2} \beta_{1}>0
\end{array}
$$

which completes the proof.

When $\tau \neq 0$, letting $1 \omega(\omega>0)$ be the root of Eq.(5), then

$$
\begin{aligned}
& C_{n} \sin \omega \tau+d \omega \cos \omega \tau=A_{n} \omega \\
& C_{n} \cos \omega \tau-d \omega \sin \omega \tau=\omega^{2}-B_{n}
\end{aligned}
$$

which leads to

$$
\omega^{4}+\left(A_{n}^{2}-2 B_{n}-d^{2}\right) \omega^{2}+B_{n}^{2}-C_{n}^{2}=0 .
$$


Let $z=\omega^{2}$, Eq. (6) becomes

$$
z^{2}+\left(A_{n}^{2}-2 B_{n}-d^{2}\right) z+B_{n}^{2}-C_{n}^{2}=0 .
$$

Through calculation we know that

$$
\begin{aligned}
A_{n}^{2}-2 B_{n}-d^{2} & =\left[\left(D_{1}+D_{2}\right) n^{2}+d-\alpha_{1}\right]^{2}-2\left[D_{1} D_{2} n^{4}+\left(D_{1} d-D_{2} \alpha_{1}\right) n^{2}-d \alpha_{1}\right]-d^{2}, \\
& =D_{2} n^{4}+2 D_{2} d n^{2}+\left(D_{1} n^{2}-\alpha_{1}\right)^{2}>0, \\
B_{n}^{2}-C_{n}^{2} & =\left(B_{n}+C_{n}\right)\left(B_{n}-C_{n}\right), \\
B_{n}+C_{n} & =D_{1} D_{2} n^{4}-D_{2} \alpha_{1} n^{2}+\alpha_{2} \beta_{1}, \\
B_{n}-C_{n} & =D_{1} D_{2} n^{4}+\left(2 D_{1} d-D_{2} \alpha_{1}\right) n^{2}-2 \alpha_{1} d-\alpha_{2} \beta_{1} .
\end{aligned}
$$

Suppose condition $(A)$ holds. It is obvious that $B_{n}+C_{n}>0$. Furthermore, if $-2 \alpha_{1} d-\alpha_{2} \beta_{1}>0$, then $B_{n}-C_{n}>0, \forall n \in$ $\mathbb{N}_{0}$; if $-2 \alpha_{1} d-\alpha_{2} \beta_{1}<0$, since $B_{0}-C_{0}=-2 \alpha_{1} d-\alpha_{2} \beta_{1}<0$, then there exists $N \in \mathbb{N}$, such that $B_{n}-C_{n}<0$ when $n<N$ and $B_{n}-C_{n}>0$ when $n>N$. So we get that if $(A)$ and $-2 \alpha_{1} d-\alpha_{2} \beta_{1}>0$ hold, then Eq. (7) has no positive root for $\forall n \in \mathbb{N}_{0}$; and if $(A)$ and $-2 \alpha_{1} d-\alpha_{2} \beta_{1}<0$ hold, Eq. (7) has a positive root

$$
z_{n}=\frac{1}{2}\left[2 B_{n}+d^{2}-A_{n}^{2}+\sqrt{\left(A_{n}^{2}-2 B_{n}-d^{2}\right)^{2}-4\left(B_{n}^{2}-C_{n}^{2}\right)}\right]
$$

when $n<N$, which means that Eq. (6) also has a positive root

$$
\omega_{n}=\frac{1}{\sqrt{2}} \sqrt{2 B_{n}+d^{2}-A_{n}^{2}+\sqrt{\left(A_{n}^{2}-2 B_{n}-d^{2}\right)^{2}-4\left(B_{n}^{2}-C_{n}^{2}\right)}}
$$

Lemma 2.3. If $(A)$ and $-2 \alpha_{1} d-\alpha_{2} \beta_{1}<0$ hold, then there exist $N \in \mathbb{N}$, such that Eq. (5) has a pair of conjugate pure imaginary roots $\pm 1 \omega_{n}$ when $\tau=\tau_{n}^{j}(n<N, j=0,1,2, \cdots)$, where

$$
\tau_{n}^{j}=\left\{\begin{array}{cl}
\frac{1}{\omega_{n}}\left(\arccos \frac{a^{*}}{c^{*}}+2 j \pi\right), & b^{*}>0 \\
\frac{1}{\omega_{n}}\left(2 \pi-\arccos \frac{a^{*}}{c^{*}}+2 j \pi\right), & b^{*}<0
\end{array}\right.
$$

here $a^{*}=\left(d A_{n}+C_{n}\right) \omega^{2}-B_{n} C_{n}, \quad b^{*}=\omega\left(A_{n} C_{n}-d B_{n}\right)-d \omega^{3}, \quad c^{*}=C_{n}^{2}+d^{2} \omega^{2}$.

Furthermore, we have Lemma 2.4. $\left.\operatorname{Re}(\mathrm{d} \lambda / \mathrm{d} \tau)\right|_{\tau=\tau_{n}^{j}}>0$

Proof. Since (5), we know that

$$
\left[2 \lambda+A_{n}-d e^{-\lambda \tau}-\tau\left(C_{n}-d \lambda\right) \lambda e^{-\lambda \tau}\right] \frac{\mathrm{d} \lambda}{\mathrm{d} \tau}=\left(C_{n}-d \lambda\right) \lambda e^{-\lambda \tau}
$$

which leads to

$$
\begin{aligned}
\left(\frac{\mathrm{d} \lambda}{\mathrm{d} \tau}\right)^{-1} & =\frac{2 \lambda+A_{n}-d e^{-\lambda \tau}}{\lambda\left(C_{n}-d \lambda\right) e^{-\lambda \tau}}-\frac{\tau}{\lambda} \\
& =\frac{2 \lambda+A_{n}}{-\lambda\left(\lambda^{2}+A_{n} \lambda+B_{n}\right)}-\frac{d}{\lambda\left(C_{n}-d \lambda\right)}-\frac{\tau}{\lambda}
\end{aligned}
$$

substituting $\tau=\tau_{n}^{j}$ into the above formula, we have

$$
\begin{aligned}
\operatorname{Re}\left(\frac{\mathrm{d} \lambda}{\mathrm{d} \tau}\right)_{\tau=\tau_{n}^{j}}^{-1} & =\operatorname{Re}\left[\frac{21 \omega_{n}+A_{n}}{-1 \omega_{n}\left(-\omega_{n}^{2}+1 \omega_{n} A_{n}+B_{n}\right)}-\frac{d}{1 \omega_{n}\left(C_{n}-1 d \omega_{n}\right)}-\frac{\tau_{n}^{j}}{1 \omega_{n}}\right] \\
& =\frac{A_{n}^{2}+2\left(\omega_{n}^{2}-B_{n}\right)}{A_{n}^{2} \omega_{n}^{2}+\left(\omega_{n}^{2}-B_{n}^{2}\right)^{2}}-\frac{d^{2}}{d^{2} \omega_{n}^{2}+C_{n}^{2}} \\
& =\frac{A_{n}^{2}-2 B_{n}-d^{2}+2 \omega_{n}^{2}}{d^{2} \omega_{n}^{2}+C_{n}^{2}}>0 .
\end{aligned}
$$

Denoting $\tau_{*}=\min \left\{\tau_{n}^{0}\right\}(0 \leq n<N)$, we come to the main conclusion for this section:

Theorem 2.5.For system (1), suppose that condition $(A)$ holds, 
(i) If $-2 \alpha_{1} d-\alpha_{2} \beta_{1}>0$, then $E_{*}\left(u_{*}, v_{*}\right)$ is locally asymptotically stable for $\forall \tau \in[0,+\infty)$;

(ii) If $-2 \alpha_{1} d-\alpha_{2} \beta_{1}<0$, then $E_{*}\left(u_{*}, v_{*}\right)$ is locally asymptotically stable when $\tau \in\left[0, \tau_{*}\right)$ and unstable when $\tau>\tau_{*}$; and system undergoes Hopf bifurcation at $E_{*}\left(u_{*}, v_{*}\right)$ when $\tau=\tau_{n}^{j}(0 \leq n<N, j=0,1,2, \cdots)$.

The bifurcating period solutions are spatially homogenous when $\tau=\tau_{0}^{j}(j=0,1,2, \cdots)$;

The bifurcating period solutions are spatially non-homogenous when $\tau=\tau_{n}^{j}(0<n<N, j=0,1,2, \cdots)$.

\section{Properties of Hopf Bifurcation}

Basing on the discussion in the previous section, we find that under some condition system (1) undergoes Hopf bifurcation, which means that system (1) has bifurcating period solutions. This section, using theory of partial differential equation from Wu [26] and Hassard [27], we study the properties of bifurcating period solutions.

Letting $\bar{u}(t, x)=u(\tau t, x), \bar{v}(t, x)=v(\tau t, x)$, and removing the " $-"$ for convenience, expanding system (2) around the origin, we get the following equation:

$$
\begin{aligned}
\frac{\partial u(t, x)}{\partial t}= & \tau D_{1} \triangle u(t, x)+\tau\left[\alpha_{1} u(t, x)-\alpha_{2} v(t, x)+\alpha_{3} u^{2}(t, x)+\alpha_{4} u(t, x) v(t, x)\right. \\
+ & \left.\alpha_{5} u^{3}(t, x)+\alpha_{6} u^{2}(t, x) v(t, x)+O(4)\right] \\
\frac{\partial v(t, x)}{\partial t}= & \tau D_{2} \triangle v(t, x)+\tau\left[-d v(t, x)+\beta_{1} u(t-\tau, x)+d v(t-\tau, x)+\beta_{2} u^{2}(t-1, x)\right. \\
& \left.+\beta_{3} u(t-1, x) v(t-1, x)+\beta_{4} u^{3}(t-1, x)+\beta_{5} u^{2}(t-1, x) v(t-1, x)+O(4)\right]
\end{aligned}
$$

where

$$
\begin{aligned}
& \alpha_{3}=-\frac{r}{k}+\frac{a v_{*}}{\left(a+u_{*}\right)^{3}}, \quad \alpha_{4}=\frac{-a}{\left(a+u_{*}\right)^{2}}, \quad \alpha_{5}=\frac{a v_{*}}{\left(a+u_{*}\right)^{4}}, \quad \alpha_{6}=\frac{-a}{\left(a+u_{*}\right)^{3}} \\
& \beta_{2}=-\frac{a \mu v_{*}}{\left(a+u_{*}\right)^{3}}, \quad \beta_{3}=\frac{a \mu}{\left(a+u_{*}\right)^{2}}, \quad \beta_{4}=\frac{a \mu v_{*}}{\left(a+u_{*}\right)^{4}}, \quad \beta_{5}=\frac{-a \mu}{\left(a+u_{*}\right)^{3}}
\end{aligned}
$$

Let

$$
u_{1}(t)=u(t, \cdot), v_{1}(t)=v(t, \cdot), U=\left(u_{1}, u_{2}\right)^{T}
$$

then system (9) can be written as the following abstract ordinary differential equation in the phase space $C=C([-1,0], X)$

$$
\frac{\mathrm{d} U(t)}{\mathrm{d} t}=\bar{\tau} D \triangle U(t)+L_{\bar{\tau}}\left(U_{t}\right)+F\left(U_{t}, \varepsilon\right)
$$

Here $L: C \mapsto X, \quad F: C \mapsto X$ are defined as

$$
L_{\varepsilon}(\phi)=\varepsilon\left(\begin{array}{c}
\alpha_{1} \phi_{1}(0)-\alpha_{2} \phi_{2}(0) \\
-d \phi_{1}(0)+\beta_{1} \phi_{1}(-1)+d \phi_{2}(-1)
\end{array}\right)
$$

and

$$
\begin{aligned}
& F(\phi, \varepsilon)=\varepsilon D \triangle \phi+L_{\varepsilon}(\phi)+f(\phi, \varepsilon) f(\phi, \varepsilon)=(\bar{\tau}+\varepsilon) \\
& \left(\begin{array}{c}
\alpha_{3} \phi_{1}^{2}(0)+\alpha_{4} \phi_{1}(0) \phi_{2}(0)+\alpha_{5} \phi_{1}^{3}(0)+\alpha_{6} \phi_{1}^{2}(0) \phi_{2}(0)+O(4) \\
\beta_{2} \phi_{1}^{2}(-1)+\beta_{3} \phi_{1}(-1) \phi_{2}(-1)+\beta_{4} \phi_{1}^{3}(-1)+\beta_{5} \phi_{1}^{2}(-1) \phi_{2}(-1)+O(4)
\end{array}\right)
\end{aligned}
$$

where $\phi=\left(\phi_{1}, \phi_{2}\right)^{T} \in C$.

Consider the following linear equation

$$
\frac{\mathrm{d} U(t)}{\mathrm{d} t}=\bar{\tau} D \triangle U(t)+L_{\bar{\tau}}\left(U_{t}\right)
$$

When $\tau=\bar{\tau}$, system (9) has pure imaginary roots, denoted as $\Lambda=\{\mathrm{i} \bar{\omega} \bar{\tau},-\mathrm{i} \bar{\omega} \bar{\tau}\}$, what's more, $\Lambda$ are also eigenvalues of the following functional differential equation.

$$
\frac{\mathrm{d} z(t)}{\mathrm{d} t}=-\bar{\tau} D n^{2} z(t)+L_{\bar{\tau}}\left(z_{t}\right)
$$


By Riesz representation theorem, there exists a $2 \times 2$ matrix $\eta^{n}(\theta, \bar{\tau})(-1 \leq \theta \leq 0)$, whose elements are of bounded variation functions, such that

$$
\begin{gathered}
-\bar{\tau} D n^{2} \phi(0)+L_{\bar{\tau}}(\phi)=\int_{-1}^{0} \mathrm{~d} \eta^{n}(\theta, \bar{\tau}) \phi(\theta) . \\
\eta^{n}(\theta, \bar{\tau})=\left\{\begin{array}{cc}
\bar{\tau}\left(\begin{array}{cc}
\alpha_{1} & -\alpha_{2} \\
0 & 0
\end{array}\right), & \theta=0 \\
0, & \theta \in(-1,0) \\
\bar{\tau}\left(\begin{array}{cc}
0 & 0 \\
\beta_{1} & d
\end{array}\right), & \theta=-1
\end{array}\right.
\end{gathered}
$$

For $\forall \phi \in \mathcal{C} \cap C^{1}$ and $\psi \in \mathcal{C} \cap C^{1}$, define the operator $A(\varepsilon)$ and $A^{*}$ respectively as follow

$$
\begin{gathered}
A(\varepsilon) \phi(\theta)= \begin{cases}\frac{\mathrm{d} \phi(\theta)}{\mathrm{d} \theta}, & \theta \in[-1,0), \\
\int_{-1}^{0} \mathrm{~d} \eta(\theta, \varepsilon) \phi(\theta), & \theta=0,\end{cases} \\
A^{*} \psi(s)=\left\{\begin{array}{l}
-\frac{\mathrm{d} \psi(s)}{\mathrm{d} s}, s \in(0,1], \\
\int_{-1}^{0} \psi(-\xi) \mathrm{d} \eta(\xi, 0), s=0 .
\end{array}\right.
\end{gathered}
$$

Define the bilinear form

$$
\begin{aligned}
(\phi, \psi) & =\psi(0) \phi(0)-\int_{-1}^{0} \int_{\xi=0}^{\theta} \psi(\xi-\theta) \mathrm{d} \eta^{n}(\theta, \bar{\tau}) \phi(\xi) \mathrm{d} \xi \\
& =\psi(0) \phi(0)+\bar{\tau} \int_{-1}^{0} \psi(\xi+1)\left(\begin{array}{cc}
0 & 0 \\
\beta_{1} & d
\end{array}\right) \phi(\xi) \mathrm{d} \xi
\end{aligned}
$$

where $\phi \in C\left([-1,0], \mathbb{R}^{2}\right), \psi \in C\left([0,1], \mathbb{R}^{*}\right)$. Then $A(0)$ and $A^{*}$ are adjoint operators, and $\pm \mathrm{i} \bar{\omega} \bar{\tau}$ are eigenvalues of $A(\bar{\tau})$ and $A^{*}$. Let $P$ and $P^{*}$ are center subspaces of $A(\bar{\tau})$ and $A^{*}$ associated with $\Lambda=\{ \pm \mathrm{i} \bar{\omega} \bar{\tau}\}$ respectively, then $P^{*}$ is the adjoint space of $P$, and $\operatorname{dim} P=\operatorname{dim} P^{*}=2$.

By a straightforward computation, we can get that

$$
p_{1}(\theta)=(1, \xi)^{T} \mathrm{e}^{\mathrm{i} \bar{\omega} \bar{\tau}}(\theta \in[-1,0]), \quad p_{2}(\theta)=\overline{p_{1}(\theta)}
$$

and

$$
q_{1}(s)=(\eta, 1) \mathrm{e}^{-\mathrm{i} \bar{\omega} \bar{\tau}}(s \in[0,1]), \quad q_{2}(s)=\overline{p_{1}(s)}
$$

are bases of $A(\bar{\tau})$ and $A^{*}$ associated with $\Lambda=\{ \pm \mathrm{i} \bar{\omega} \bar{\tau}\}$, where

$$
\begin{aligned}
& \xi=\frac{1}{\alpha_{2}}\left(\alpha_{1}-D_{1} n^{2}-\mathrm{i} \bar{\omega}\right), \\
& \eta=\frac{1}{\alpha_{2}}\left[-d-D_{2} n^{2}+d \cos \bar{\omega} \bar{\tau}+\mathrm{i}(\bar{\omega}+d \sin \bar{\omega} \bar{\tau})\right] .
\end{aligned}
$$

Taking

$$
\begin{aligned}
& \Phi_{1}(\theta)=\frac{1}{2}\left[p_{1}(\theta)+p_{2}(\theta)\right]=\left(\begin{array}{c}
\operatorname{Re}\left(\mathrm{e}^{\mathrm{i} \bar{\omega} \bar{\tau} \theta}\right) \\
\operatorname{Re}\left(\xi \mathrm{e}^{\mathrm{i} \bar{\omega} \bar{\tau} \theta}\right)
\end{array}\right) \\
& =\left(\begin{array}{c}
\cos (\bar{\omega} \bar{\tau} \theta) \\
\frac{1}{\alpha_{2}}\left[\left(\alpha_{1}-D_{1} n^{2}\right) \cos (\bar{\omega} \bar{\tau} \theta)+\bar{\omega} \sin (\bar{\omega} \bar{\tau} \theta)\right]
\end{array}\right) \\
& \Phi_{2}(\theta)=\frac{1}{2}\left[p_{1}(\theta)-p_{2}(\theta)\right]=\left(\begin{array}{c}
\operatorname{Im}\left(\mathrm{e}^{\mathrm{i} \bar{\omega} \bar{\tau} \theta}\right) \\
\operatorname{Im}\left(\xi \mathrm{e}^{\mathrm{i} \bar{\omega} \bar{\tau} \theta}\right)
\end{array}\right) \\
& =\left(\begin{array}{c}
\sin (\bar{\omega} \bar{\tau} \theta) \\
\frac{1}{\alpha_{2}}\left[\left(\alpha_{1}-D_{1} n^{2}\right) \sin (\bar{\omega} \bar{\tau} \theta)-\bar{\omega} \cos (\bar{\omega} \bar{\tau} \theta)\right]
\end{array}\right)
\end{aligned}
$$


and

$$
\begin{aligned}
\Psi_{1}^{*}(s) & =\frac{1}{2}\left[q_{1}(s)+q_{2}(s)\right]=\left(\operatorname{Re}\left(\eta \mathrm{e}^{-\mathrm{i} \bar{\omega} \bar{\tau} \theta}\right), \operatorname{Re}\left(\mathrm{e}^{-\mathrm{i} \bar{\omega} \bar{\tau} \theta}\right)\right) \\
& =\left(\frac{1}{\alpha_{2}}\left[\left(-d-D_{2} n^{2}+d \cos (\bar{\omega} \bar{\tau} s)\right) \cos (\bar{\omega} \bar{\tau} s)+(\bar{\omega}+d \sin (\bar{\omega} \bar{\tau} s)) \sin (\bar{\omega} \bar{\tau} s)\right], \cos (\bar{\omega} \bar{\tau} s)\right) \\
\Psi_{2}^{*}(s) & =\frac{1}{2}\left[q_{1}(s)-q_{2}(s)\right]=\left(\operatorname{Im}\left(\eta \mathrm{e}^{-\mathrm{i} \bar{\omega} \bar{\tau} s}\right), \operatorname{Im}\left(\mathrm{e}^{-\mathrm{i} \bar{\omega} \bar{\tau} s}\right)\right) \\
& =\left(\frac{1}{\alpha_{2}}\left[\left(d+D_{2} n^{2}-d \cos (\bar{\omega} \bar{\tau} s)\right) \sin (\bar{\omega} \bar{\tau} s)+(\bar{\omega}+d \sin (\bar{\omega} \bar{\tau} s)) \cos (\bar{\omega} \bar{\tau} s)\right],-\sin (\bar{\omega} \bar{\tau} s)\right)
\end{aligned}
$$

then $\left(\Phi_{1}, \Phi_{2}\right)$ and $\left(\Psi_{1}^{*}, \Psi_{2}^{*}\right)$ are bases for $P$ and $P^{*}$ respectively.

By (14), we have

$$
\begin{aligned}
& M_{1}:=\left(\Psi_{1}^{*}, \Phi_{1}\right)=\frac{1}{2 \alpha_{2}}[2 \gamma+\bar{\tau} \gamma \rho-d \bar{\omega} \bar{\tau} \sin (\bar{\omega} \bar{\tau})] \\
& M_{2}:=\left(\Psi_{1}^{*}, \Phi_{2}\right)=\frac{-1}{2 \alpha_{2}}[2 \bar{\omega}+d \bar{\omega} \bar{\tau} \rho+\bar{\tau} \gamma \sin (\bar{\omega} \bar{\tau})] \\
& M_{3}:=\left(\Psi_{2}^{*}, \Phi_{1}\right)=\frac{1}{2 \alpha_{2}}[2 \bar{\omega}-d \bar{\omega} \bar{\tau} \delta-\bar{\tau} \gamma \sin (\bar{\omega} \bar{\tau})] \\
& M_{4}:=\left(\Psi_{2}^{*}, \Phi_{2}\right)=\frac{1}{2 \alpha_{2}}[\bar{\tau} \gamma \delta-d \bar{\omega} \bar{\tau} \sin (\bar{\omega} \bar{\tau})]
\end{aligned}
$$

where

$$
\gamma=\alpha_{1}-D_{1} n^{2}-D_{2} n^{2}, \quad \rho=\cos (\bar{\omega} \bar{\tau})+\frac{\sin (\bar{\omega} \bar{\tau})}{\bar{\omega} \bar{\tau}}, \quad \delta=\cos (\bar{\omega} \bar{\tau})-\frac{\sin (\bar{\omega} \bar{\tau})}{\bar{\omega} \bar{\tau}} .
$$

Define

$$
(\Psi, \Phi)=\left(\Psi_{j}^{*}, \Phi_{k}\right)=\left(\begin{array}{cc}
\left(\Psi_{1}^{*}, \Phi_{1}\right) & \left(\Psi_{1}^{*}, \Phi_{2}\right) \\
\left(\Psi_{2}^{*}, \Phi_{1}\right) & \left(\Psi_{2}^{*}, \Phi_{2}\right)
\end{array}\right)
$$

and choose $\Psi=\left(\Psi_{1}, \Psi_{2}\right)^{T}=\left(\Psi^{*}, \Phi\right)^{-1} \Psi^{*}$, then we have $(\Psi, \Phi)=I_{2}$, and

$$
\begin{aligned}
\Psi_{1}(0)-\mathrm{i} \Psi_{2}(0) & =\frac{1}{M_{1} M_{4}-M_{2} M_{3}}\left(\frac{-D_{2} n^{2}}{\alpha_{2}}\left(M_{3}+M_{4} \mathrm{i}\right)-\bar{\omega}\left(M_{2}+M_{1} \mathrm{i}\right), M_{4}+M_{3} \mathrm{i}\right) \\
& :=\left(\Upsilon_{1}, \Upsilon_{2}\right)
\end{aligned}
$$

Take $f_{n}=\left(\beta_{n}^{1}, \beta_{n}^{2}\right)$, here

$$
\beta_{n}^{1}=\left(\begin{array}{c}
\cos (n x) \\
0
\end{array}\right), \quad \beta_{n}^{2}=\left(\begin{array}{c}
0 \\
\cos (n x)
\end{array}\right)
$$

and denote

$$
c \cdot f_{n}:=c_{1} \beta_{n}^{1}+c_{2} \beta_{n}^{2}, \quad c=\left(c_{1}, c_{2}\right)^{T} \in C([-1,0], X)
$$

For $u=\left(u_{1}, u_{2}\right), v=\left(v_{1}, v_{2}\right), u, v \in X$, define inner product as

$$
\langle u, v\rangle=\frac{1}{\pi} \int_{0}^{\pi} u_{1} \bar{v}_{1} \mathrm{~d} x+\frac{1}{\pi} \int_{0}^{\pi} u_{2} \bar{v}_{2} \mathrm{~d} x .
$$

For $\phi \in C=C([-1,0], X)$, define $\left\langle\phi, f_{n}\right\rangle=\left(\left\langle\phi, f_{n}^{1}\right\rangle,\left\langle\phi, f_{n}^{2}\right\rangle\right)^{T}$, then we get the center subspace of Eq. (12)

$$
P_{C N} C(\phi)=\Phi\left(\Psi,\left\langle\phi, f_{k}\right\rangle\right) \cdot f_{k}, \phi \in C,
$$

and we can decompose space into $C=P_{C N} C \oplus P_{S} C$, where $P_{S} C$ is the complementary space of $P_{C N} C$ in $C$.

Let $A_{\bar{\tau}}$ be the infinitesimal generator of the semigroup induced by solutions of Eq. (13), then (2) can be written as the abstract ODE

$$
\frac{\mathrm{d} U(t)}{\mathrm{d} t}=A_{\bar{\tau}} U_{t}+R\left(U_{t}, \varepsilon\right)
$$

where

$$
R\left(U_{t}, \varepsilon\right)=\left\{\begin{array}{cc}
0, & \theta \in[-1,0) \\
F\left(U_{t}, \varepsilon\right), & \theta=0
\end{array}\right.
$$


We can decompose the abstract equation into

$$
U_{t}=\Phi\left(\begin{array}{c}
x_{1} \\
x_{2}
\end{array}\right) \cdot f_{n}+h\left(x_{1}, x_{2}, \varepsilon\right)
$$

where

$$
\left(\begin{array}{l}
x_{1} \\
x_{2}
\end{array}\right)=\left(\Psi,\left\langle U_{t}, f_{n}\right\rangle\right), h\left(x_{1}, x_{2}, \varepsilon\right) \in P_{S} C, \quad h(0,0,0)=0, \quad \operatorname{Dh}(0,0,0)=0 .
$$

Solutions of (10) on the center manifold are

$$
U_{t}=\Phi\left(\begin{array}{l}
x_{1}(t) \\
x_{2}(t)
\end{array}\right) \cdot f_{n}+h\left(x_{1}, x_{2}, 0\right)
$$

Let $z(t)=x_{1}(t)-\mathrm{i} x_{2}(t)$, then we have

$$
\dot{z}(t)=\mathrm{i} \bar{\omega} \bar{\tau} z(t)+g(z, \bar{z})
$$

where

$$
g(z, \bar{z})=\left(\Psi_{1}(0)-\mathrm{i} \Psi_{2}(0)\right)\left\langle F\left(U_{t}, 0\right), f_{n}\right\rangle
$$

Substitute $z(t)$ into (17), we get

$$
\Phi\left(\begin{array}{l}
x_{1}(t) \\
x_{2}(t)
\end{array}\right) \cdot f_{n}=\left(\Phi_{1}, \Phi_{2}\right)\left(\begin{array}{c}
\frac{z+\bar{z}}{2} \\
\frac{\mathrm{i}(z-\bar{z})}{2}
\end{array}\right) \cdot f_{n}=\frac{1}{2}\left(p_{1} z+\bar{p}_{1} \bar{z}\right) \cdot f_{n}
$$

and

$$
h\left(x_{1}, x_{2}, 0\right)=h\left(\frac{z+\bar{z}}{2}, \frac{\mathrm{i}(z-\bar{z})}{2}, 0\right)
$$

then $U_{t}$ can be changed into the following form

$$
U_{t}=\frac{1}{2}\left(p_{1} z+\bar{p}_{1} \bar{z}\right) \cdot f_{n}+h\left(\frac{z+\bar{z}}{2}, \frac{\mathrm{i}(z-\bar{z})}{2}, 0\right):=\frac{1}{2}\left(p_{1} z+\bar{p}_{1} \bar{z}\right) \cdot f_{n}+W(z, \bar{z})
$$

Let

$$
W(z, \bar{z})=W_{20} \frac{z^{2}}{2}+W_{11} z \bar{z}+W_{02} \frac{\bar{z}^{2}}{2}+\cdots, g(z, \bar{z}) \quad=g_{20} \frac{z^{2}}{2}+g_{11} z \bar{z}+g_{02} \frac{\bar{z}^{2}}{2}+\cdots
$$

then by (11) and (18) we get

$$
\begin{aligned}
\left\langle F\left(U_{t}, 0\right), f_{n}\right\rangle= & \frac{\bar{\tau} z^{2}}{4}\left(\begin{array}{c}
\alpha_{3}+\alpha_{4} \xi \\
\mathrm{e}^{-2 \mathrm{i} \overline{\bar{\tau}}}\left(\beta_{2}+\beta_{3} \xi\right)
\end{array}\right) \frac{1}{\pi} \int_{0}^{\pi} \cos ^{3}(n x) \mathrm{d} x \\
+ & \frac{\bar{\tau} z \bar{z}}{4}\left(\begin{array}{c}
2 \alpha_{3}+\alpha_{4}(\xi+\bar{\xi}) \\
2 \beta_{2}+\beta_{3}(\xi+\bar{\xi})
\end{array}\right) \frac{1}{\pi} \int_{0}^{\pi} \cos ^{3}(n x) \mathrm{d} x \\
+ & \frac{\bar{\tau} \bar{z}^{2}}{4}\left(\begin{array}{c}
\alpha_{3}+\alpha_{4} \bar{\xi} \\
\mathrm{e}^{2 \mathrm{i} \bar{\omega} \bar{\tau}}\left(\beta_{2}+\beta_{3} \bar{\xi}\right)
\end{array}\right) \frac{1}{\pi} \int_{0}^{\pi} \cos ^{3}(n x) \mathrm{d} x \\
+\bar{\tau} z^{2} \bar{z} & \left(\begin{array}{l}
\alpha_{3}\left\langle\left(W_{11}^{(1)}(0)+\frac{1}{2} W_{20}^{(1)}(0)\right) \cos (n x), \cos (n x)\right\rangle \\
+\alpha_{4}\left\langle\left(\frac{1}{2} W_{11}^{(2)}(0)+\frac{1}{4} W_{20}^{(2)}(0)+\frac{\xi}{4} W_{20}^{(1)}(0)+\frac{\xi}{2} W_{11}^{(1)}(0)\right) \cos (n x), \cos (n x)\right\rangle \\
+\frac{1}{8}\left(3 \alpha_{3}+\alpha_{6} \bar{\xi}+2 \alpha_{6} \xi\right)\left\langle\cos ^{3}(n x), \cos (n x)\right\rangle \\
\beta_{2}\left\langle\left(\mathrm{e}^{-\mathrm{i} \bar{\omega} \bar{\tau}} W_{11}^{(1)}(-1)+\frac{\mathrm{e}^{\mathrm{i} \bar{\omega} \bar{\tau}}}{2} W_{20}^{(1)}(-1)\right) \cos (n x), \cos (n x)\right\rangle \\
+\beta_{3}\left\langle\left(\frac{\mathrm{e}^{-\mathrm{i} \bar{\omega} \bar{\tau}}}{2}\left(W_{11}^{(2)}(-1)+\xi W_{11}^{(1)}(-1)\right)\right) \cos (n x), \cos (n x)\right\rangle \\
+\beta_{3}\left\langle\left(\frac{\mathrm{e}^{\mathrm{i} \bar{\omega} \bar{\tau}}}{4}\left(W_{20}^{(2)}(-1)+\bar{\xi} W_{20}^{(1)}(-1)\right)\right) \cos (n x), \cos (n x)\right\rangle \\
+\frac{\mathrm{e}^{-\mathrm{i} \bar{\omega} \bar{\tau}}}{8}\left(3 \beta_{4}+\beta_{5} \bar{\xi}+2 \beta_{5} \xi\right)\left\langle\cos ^{3}(n x), \cos (n x)\right\rangle
\end{array}\right.
\end{aligned}
$$


Since $\int_{0}^{\pi} \cos ^{3}(n x) \mathrm{d} x=0$ when $n \neq 0$, combining with (15), we have

$$
\begin{aligned}
& g_{20}=\overline{g_{02}}=\left\{\begin{array}{cc}
0, & n \neq 0 \\
\bar{\tau}\left[\Upsilon_{1}\left(\alpha_{3}+\xi \alpha_{4}\right)+\Upsilon_{2} \mathrm{e}^{-2 \mathrm{i} \bar{\omega} \bar{\tau}}\left(\beta_{2}+\xi \beta_{3}\right)\right], & n=0
\end{array}\right.
\end{aligned}
$$

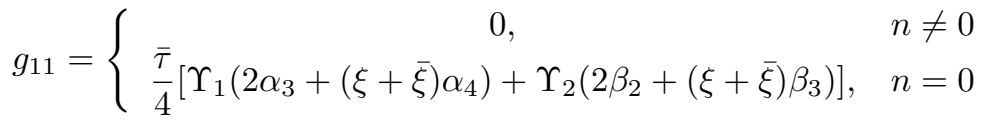

$$
\begin{aligned}
& g_{21}=\bar{\tau} \Upsilon_{1}\left(\begin{array}{l}
\alpha_{3}\left\langle\left(2 W_{11}^{(1)}(0)+W_{20}^{(1)}(0)\right) \cos (n x), \cos (n x)\right\rangle \\
\left.+\alpha_{4}\left\langle\left(W_{11}^{(2)}(0)+\frac{1}{2} W_{20}^{(2)}(0)+\frac{\bar{\xi}}{2} W_{20}^{(1)}(0)+\xi W_{11}^{(1)}(0)\right) \cos (n x), \cos (n x)\right\rangle\right) \\
+\frac{1}{4}\left(3 \alpha_{3}+\alpha_{6} \bar{\xi}+2 \alpha_{6} \xi\right)\left\langle\cos ^{3}(n x), \cos (n x)\right\rangle
\end{array}\right) \\
& +\bar{\tau} \Upsilon_{2}\left(\begin{array}{l}
\beta_{2}\left\langle\left(2 \mathrm{e}^{-\mathrm{i} \bar{\omega} \bar{\tau}} W_{11}^{(1)}(-1)+\mathrm{e}^{\mathrm{i} \bar{\omega} \bar{\tau}} W_{20}^{(1)}(-1)\right) \cos (n x), \cos (n x)\right\rangle \\
+\beta_{3}\left\langle\left(\mathrm{e}^{-\mathrm{i} \bar{\omega} \bar{\tau}}\left(W_{11}^{(2)}(-1)+\xi W_{11}^{(1)}(-1)\right)\right) \cos (n x), \cos (n x)\right\rangle \\
+\beta_{3}\left\langle\left(\frac{\mathrm{e}^{\mathrm{i} \bar{\omega} \bar{\tau}}}{2}\left(W_{20}^{(2)}(-1)+\bar{\xi} W_{20}^{(1)}(-1)\right)\right) \cos (n x), \cos (n x)\right\rangle \\
+\frac{\mathrm{e}^{-\mathrm{i} \bar{\omega} \bar{\tau}}}{4}\left(3 \beta_{4}+\beta_{5} \bar{\xi}+2 \beta_{5} \xi\right)\left\langle\cos ^{3}(n x), \cos (n x)\right\rangle
\end{array}\right)
\end{aligned}
$$

Here we need to compute $W_{20}(\theta), W_{11}(\theta), \quad \theta \in[-1,0]$.

By (18)

$$
\begin{aligned}
\dot{W}(z, \bar{z}) & =W_{20} z \dot{z}+W_{11} \dot{z} \bar{z}+W_{11} z \dot{\bar{z}}+W_{02} \bar{z} \dot{\bar{z}}+\cdots \\
& :=A_{\bar{\tau}} W+H(z, \bar{z}) \\
A_{\bar{\tau}} W(z, \bar{z}) & =A_{\bar{\tau}} W_{20} \frac{z^{2}}{2}+A_{\bar{\tau}} W_{11} z \bar{z}+A_{\bar{\tau}} W_{02} \frac{\bar{z}^{2}}{2}+\cdots
\end{aligned}
$$

here

$$
\begin{aligned}
H(z, \bar{z}) & =H_{20} \frac{z^{2}}{2}+H_{11} z \bar{z}+H_{02} \frac{\bar{z}^{2}}{2}+\cdots \\
& =X_{0} F\left(U_{t}, 0\right)-\Phi\left(\Psi,\left\langle X_{0} F\left(U_{t}, 0\right), f_{n}\right\rangle\right) \cdot f_{n}
\end{aligned}
$$

so

$$
\left(2 \mathrm{i} \bar{\omega} \bar{\tau}-A_{\bar{\tau}}\right) W_{20}=H_{20}, \quad-A_{\bar{\tau}} W_{11}=H_{11}, \quad\left(-2 \mathrm{i} \bar{\omega} \bar{\tau}-A_{\bar{\tau}}\right) W_{02}=H_{02},
$$

here we need to find out $H_{20}(\theta)$ and $H_{11}(\theta)$.

When $\theta \in[-1,0)$, we know that

$$
\begin{aligned}
& H(z, \bar{z})=-\Phi(\theta) \Psi(0)\left\langle F\left(U_{t}, 0\right), f_{n}\right\rangle \cdot f_{n} \\
& =-\left(\frac{p_{1}(\theta)+p_{2}(\theta)}{2}, \frac{p_{1}(\theta)-p_{2}(\theta)}{2 \mathrm{i}}\right)\left(\begin{array}{c}
\Psi_{1}(0) \\
\Psi_{2}(0)
\end{array}\right)\left\langle F\left(U_{t}, 0\right), f_{n}\right\rangle \cdot f_{n} \\
& =-\frac{1}{2}\left[p_{1}(\theta)\left(\Psi_{1}(0)-\mathrm{i} \Psi_{2}(0)\right)+p_{2}(\theta)\left(\Psi_{(}(0)+\mathrm{i} \Psi_{2}(0)\right)\right]\left\langle F\left(U_{t}, 0\right), f_{n}\right\rangle \cdot f_{n} \\
& =-\frac{1}{2}\left(p_{1}(\theta) g_{20}+p_{2}(\theta) \bar{g}_{02}\right) \cdot f_{n} \frac{z^{2}}{2}-\frac{1}{2}\left(p_{1}(\theta) g_{11}+p_{2}(\theta) \bar{g}_{11}\right) \cdot f_{n} \frac{z^{2}}{2}+\cdots \\
& -\frac{1}{2}\left(p_{1}(\theta) g_{02}+p_{2}(\theta) \bar{g}_{20}\right) \cdot f_{n} \frac{\bar{z}^{2}}{2}
\end{aligned}
$$

so we have

$$
\begin{aligned}
& H_{20}(\theta)=\left\{\begin{array}{cc}
0, & n=1,2,3, \cdots \\
-\frac{1}{2}\left(p_{1}(\theta) g_{20}+p_{2}(\theta) \bar{g}_{02}\right) \cdot f_{0}, & n=0
\end{array}\right. \\
& H_{11}(\theta)=\left\{\begin{array}{cc}
0, & n=1,2,3, \cdots \\
-\frac{1}{2}\left(p_{1}(\theta) g_{11}+p_{2}(\theta) \bar{g}_{11}\right) \cdot f_{0}, & n=0
\end{array}\right. \\
& H_{02}(\theta)=\left\{\begin{array}{cc}
0, & n=1,2,3, \cdots \\
-\frac{1}{2}\left(p_{1}(\theta) g_{02}+p_{2}(\theta) \bar{g}_{20}\right) \cdot f_{0}, & n=0
\end{array}\right.
\end{aligned}
$$


when $\theta=0, H(z, \bar{z})(0)=F\left(U_{t}, 0\right)-\Phi\left(\Psi,\left\langle F\left(U_{t}, 0\right), f_{n}\right\rangle\right) \cdot f_{n}$, so we have

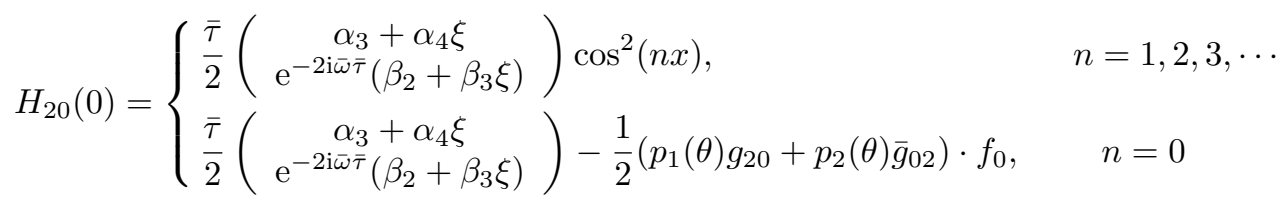

$$
\begin{aligned}
& H_{11}(0)=\left\{\begin{array}{lc}
\bar{\tau}\left(\begin{array}{c}
2 \alpha_{3}+\alpha_{4}(\xi+\bar{\xi}) \\
2 \beta_{2}+\beta_{3}(\xi+\bar{\xi})
\end{array}\right) \cos ^{2}(n x), & n=1,2,3, \cdots \\
\bar{\tau}\left(\begin{array}{c}
2 \alpha_{3}+\alpha_{4}(\xi+\bar{\xi}) \\
2 \beta_{2}+\beta_{3}(\xi+\bar{\xi})
\end{array}\right)-\frac{1}{2}\left(p_{1}(\theta) g_{11}+p_{2}(\theta) \bar{g}_{11}\right) \cdot f_{0}, & n=0
\end{array}\right.
\end{aligned}
$$

By the definition of $A_{\bar{\tau}}$ and (19),we have

$$
\dot{W}_{20}=A_{\bar{\tau}} W_{20}=2 \mathrm{i} \bar{\omega} \bar{\tau} W_{20}+\frac{1}{2}\left(p_{1} g_{20}+p_{2} \bar{g}_{02}\right) \cdot f_{n}, \quad-1 \leq \theta<0
$$

therefore

$$
W_{20}(\theta)=\frac{\mathrm{i}}{2 \bar{\omega} \bar{\tau}}\left(g_{20} p_{1}(\theta)+\frac{\bar{g}_{02}}{3} p_{2}(\theta)\right) \cdot f_{n}+E_{1} \mathrm{e}^{2 \mathrm{i} \bar{\omega} \bar{\tau} \theta}
$$

where

$$
E_{1}=\left\{\begin{array}{lc}
W_{20}(0), & n=1,2,3, \cdots \\
W_{20}(0)-\frac{\mathrm{i}}{2 \bar{\omega} \bar{\tau}}\left(g_{20} p_{1}(0)+\frac{\bar{g}_{02}}{3} p_{2}(0)\right) \cdot f_{0}, & n=0
\end{array}\right.
$$

furthermore, by the definition of $A_{\bar{\tau}}$, we have

$$
\begin{aligned}
2 \mathrm{i} \bar{\omega} \bar{\tau} & {\left[\frac{\mathrm{i}}{2 \bar{\omega} \bar{\tau}}\left(g_{20} p_{1}(0)+\frac{\bar{g}_{02}}{3} p_{2}(0)\right) \cdot f_{0}+E_{1}\right]-\bar{\tau} D \triangle\left[\frac{\mathrm{i}}{2 \bar{\omega} \bar{\tau}}\left(g_{20} p_{1}(0)+\frac{\bar{g}_{02}}{3} p_{2}(0)\right) \cdot f_{0}+E_{1}\right] } \\
& -L(\bar{\tau})\left[\frac{\mathrm{i}}{2 \bar{\omega} \bar{\tau}}\left(g_{20} p_{1}(\theta)+\frac{\bar{g}_{02}}{3} p_{2}(\theta)\right) \cdot f_{0}+E_{1} \mathrm{e}^{2 \mathrm{i} \bar{\omega} \bar{\tau}}\right] \\
= & \frac{\bar{\tau}}{2}\left(\begin{array}{c}
\alpha_{3}+\alpha_{4} \xi \\
\mathrm{e}^{-2 \mathrm{i} \bar{\tau}}\left(\beta_{2}+\beta_{3} \xi\right)
\end{array}\right)-\frac{1}{2}\left(p_{1}(\theta) g_{20}+p_{2}(\theta) \bar{g}_{02}\right) \cdot f_{0}
\end{aligned}
$$

notice that

$$
\begin{aligned}
& \bar{\tau} D \triangle\left[p_{1}(0) \cdot f_{0}\right]+L(\bar{\tau})\left[p_{1}(\theta) \cdot f_{0}\right]=\mathrm{i} \bar{\omega} \bar{\tau} p_{1}(0) \cdot f_{0} \\
& \bar{\tau} D \triangle\left[p_{2}(0) \cdot f_{0}\right]+L(\bar{\tau})\left[p_{2}(\theta) \cdot f_{0}\right]=-\mathrm{i} \bar{\omega} \bar{\tau} p_{2}(0) \cdot f_{0}
\end{aligned}
$$

we get

$$
2 \mathrm{i} \bar{\omega} \bar{\tau} E_{1}-\bar{\tau} D \triangle E_{1}-L(\bar{\tau})\left(E_{1} \mathrm{e}^{2 \mathrm{i} \bar{\omega} \bar{\tau} \theta}\right)=\frac{\bar{\tau}}{2}\left(\begin{array}{c}
\alpha_{3}+\alpha_{4} \xi \\
\mathrm{e}^{-2 \mathrm{i} \bar{\omega} \bar{\tau}}\left(\beta_{2}+\beta_{3} \xi\right)
\end{array}\right) \cos ^{2}(n x)
$$

and

$$
E_{1}=\left(\begin{array}{cc}
2 \mathrm{i} \bar{\omega}+D_{1} n^{2}-\alpha_{1} & \alpha_{2} \\
-\beta_{1} \mathrm{e}^{-2 \mathrm{i} \bar{\omega} \bar{\tau}} & 2 \mathrm{i} \bar{\omega}+D_{2} n^{2}+d-d \mathrm{e}^{-2 \mathrm{i} \bar{\omega} \bar{\tau}}
\end{array}\right)^{-1}\left(\begin{array}{c}
\alpha_{3}+\alpha_{4} \xi \\
\mathrm{e}^{-2 \mathrm{i} \bar{\omega} \bar{\tau}}\left(\beta_{2}+\beta_{3} \xi\right)
\end{array}\right) \frac{\cos ^{2}(n x)}{2}
$$

Since

$$
\dot{W}_{11}(\theta)=\frac{1}{2}\left[p_{1}(\theta) g_{11}+p_{2}(\theta) \bar{g}_{11}\right] \cdot f_{n},
$$

then

$$
W_{11}(\theta)=\frac{\mathrm{i}}{2 \bar{\omega} \bar{\tau}}\left(p_{2}(\theta) \bar{g}_{11}-p_{1}(\theta) g_{11}\right)+E_{2}
$$

similarly we get

$$
E_{2}=\left(\begin{array}{cc}
D_{1} n^{2}-\alpha_{1} & \alpha_{2} \\
-\beta_{1} & D_{2} n^{2}
\end{array}\right)^{-1}\left(\begin{array}{c}
2 \alpha_{3}+\alpha_{4}(\xi+\bar{\xi}) \\
2 \beta_{2}+\beta_{3}(\xi+\bar{\xi})
\end{array}\right) \frac{\cos ^{2}(n x)}{4}
$$


Therefor $g_{21}$ can be expressed by the parameters in system (1). Thus, we can compute the following quantities:

$$
\begin{aligned}
c_{1}(0) & =\frac{\mathrm{i}}{2 \bar{\omega} \bar{\tau}}\left(g_{20} g_{11}-2\left|g_{11}\right|^{2}-\frac{1}{3}\left|g_{02}\right|^{2}\right)+\frac{g_{21}}{2} \\
\mu_{2} & =-\frac{\operatorname{Re} c_{1}(0)}{\operatorname{Re} \lambda^{\prime}(\bar{\tau})} \\
\beta_{2} & =2 \operatorname{Re} c_{1}(0) \\
T_{2} & =-\frac{\operatorname{Im} c_{1}(0)+\mu_{2} \operatorname{Im} \lambda^{\prime}(\bar{\tau})}{\bar{\omega} \bar{\tau}}
\end{aligned}
$$

which determine the properties of bifurcating periodic solutions at the critical value $\tau_{0}$. The direction and stability of Hopf bifurcation in the center manifold can be determined by $\mu_{2}$ and $\beta_{2}$ respectively. In fact, if $\mu_{2}>0\left(\mu_{2}<0\right)$, then the bifurcating periodic solutions are forward (backward); the bifurcating periodic solutions on the center manifold are stable (unstable) if $\beta_{2}<0\left(\beta_{2}>0\right)$; and $T_{2}$ determines

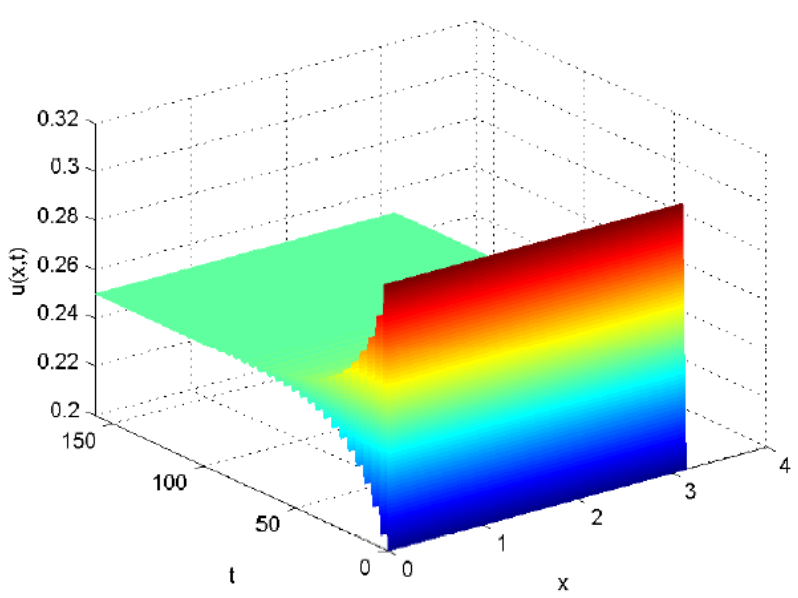

Figure 1. When $\tau=0.25<\tau_{0}$, the positive equilibrium of system (1) is asymptotically stable with parameters given in (a), and the initial value is $(0.3,9)$.
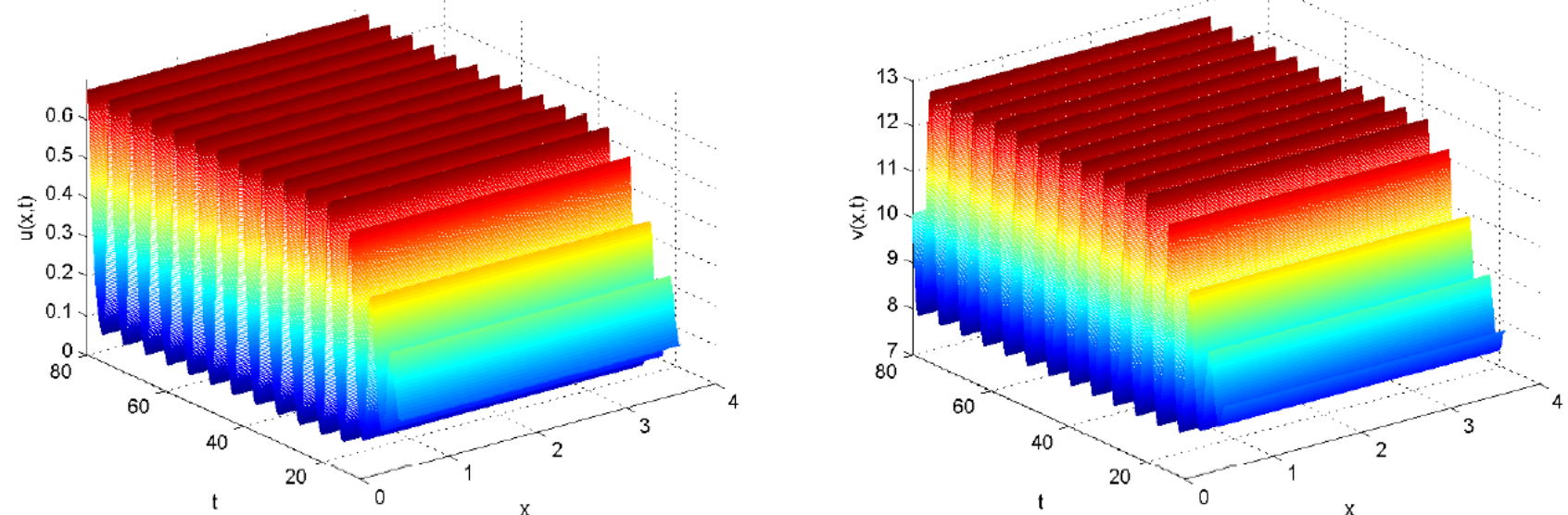

Figure 2. When $\tau=0.4>\tau_{0}$, the spatial homogeneous periodic solution bifurcating from the positive equilibrium of system (1) with parameters given in (a), and the initial value is $(0.3,9)$. the period of the bifurcating periodic solutions: the period increases (decreases) if $T_{2}>0\left(T_{2}<0\right)$.

\section{Numerical Simulations}

We choose a set of parameters as follows:

$$
\begin{aligned}
\text { (a) } & a=1, \quad b=0.4, d=0.4, k \\
& =4, \\
r & =6, \mu=2, D_{1}=2, \quad D_{2}=0.5
\end{aligned}
$$

then $E_{0}=(4.06559,0), \quad E_{*}=(0.25,9.03125)$. Basing on the analysis previously, we get

$$
B_{0}-C_{0}<0, B_{n}-C_{n}>0(n \geq 1)
$$

which means that Hopf bifurcation occurs only when $n=$ 0 and $\tau_{0}^{0}=0.2727$.

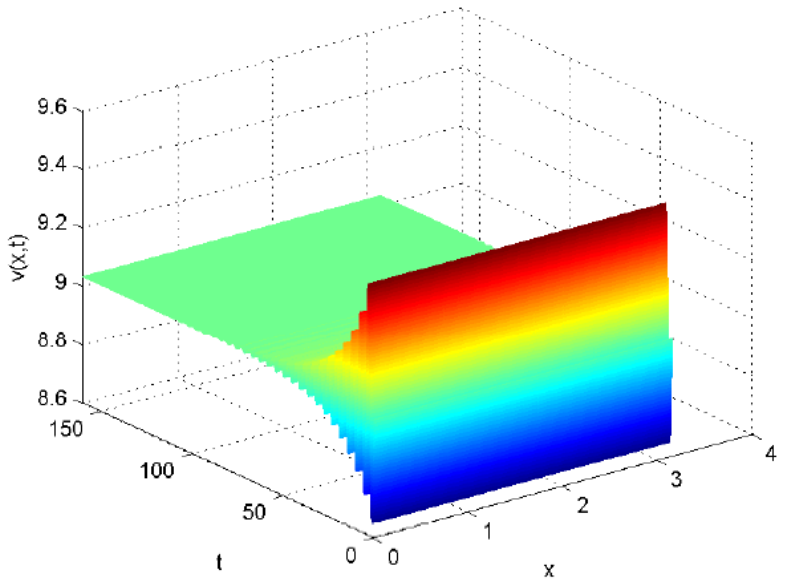




\section{Conclusion}

In this paper, we consider the diffusive RosenzweigMacArthur model with a constant delay $\tau$ due to the gestation of predator. We find that if condition (A) holds, then stability of the positive equilibrium $E_{*}\left(u_{*}, v_{*}\right)$ was influenced by the choose of the parameters. With the parameter set $(a)$ as shown in section 4 , we find that $E_{*}\left(u_{*}, v_{*}\right)$ loses its stability and an spatial homogenous orbitally asymptotically stable periodic solution arises from the Hopf bifurcation when the delay $\tau$ passes through some critical value $\tau_{0}^{0}$. This shows the important influence of the time delay $\tau$ to the system.

\section{Acknowledgements}

Thanks a lot for the kindly comments and suggestions from the reviewers and the handling editor, which led to a great improvement in the presentation of this work. In addition, this work is supported by the Doctor Foundation of Harbin University (HUDF2014-010).

\section{References}

[1] V. A. A. Jansen, The dynamics of two diffusively coupled predator-prey populations, Theoretical Population Biology, 59 (2001) 119-131.

[2] Y. Kuang, Delay differential Equation with Applications in Population Dynamics, Academic Press, New York, 1993.

[3] C. S. Holling, The functional response of predator to prey density and its role in minicry and population regulation, Mem. Ent. Sec. Can., 45 (1965) 1-60.

[4] X. Liu, L. Chen, Complex dynamics of Holling type II Lotka-Volterra predator-prey system with impulsive perturbations on the predator, Chaos, Solitons \& Fractals, 16 (2003) 311-320.

[5] E. Beretta, Y. Kuang, Global analyses in some delayed ratio-dependent predator-prey systems, Nonlinear Ana. TMA., 32 (1998) 381-408.

[6] W. Ko, k. Ryu, Qualitative analysis of a predatorprey model with Holling type II functional response incorporating a prey fefuge, J. Differential Equations, 231(2006) 534-550.

[7] M. Rosinzweig and R. MacArthur, Graphical representation and stability conditions of predator-prey interation, American Naturlist, 97 (1963) 209-223.

[8] S. B. Hsu, On global stability of a predator-prey system. Math. Biosci., 39 (1978) 1C10.

[9] S. B. Hsu, P. Waltman, Competing predators, SIAM J. Math. Anal., 35 (1978) 617-625.
[10] S. B. Hsu, T. W. Hwang and Y. Kuang,Global analysis of the Michaelis-Menten-type ratio-dependent predatorprey system. J. Math. Biol., 42 (1978) 489C506.

[11] D. Xiao, S. Ruan, Global dynamics of a ratio-dependent predator-prey system, J. Math. Biol., 43 (2001)268-290.

[12] X. Tian, R. Xu, Global dynamics of a predatorprey system with Holling type II functional response, Nonlinear Analysis: Modelling and Control., 16 (2011) 242-253.

[13] K. Cheng, Uniqueness of a limit cycle for a predator-prey system, SIAM J. Math. Anal., 12 (1981), 541-548.

[14] Y. Kuang, H. I. Freedman, Uniqueness of limit cycles in Gause-type models of predator-prey systems, Math. Biosci., 88 (1988) 67-84.

[15] D. Xiao, Z. Zhang, On the uniqueness and nonexistence of limit cycles for predator-prey system,Nonlinearity, 16 (2003) 1-17.

[16] R. Xu, M. A. J. Chaplain, F.A. Davidson, Periodic solutons for a predator-prey model with Holling type II functional response and time delays Appl. Math. Comput., 161 (2005) 637-654.

[17] M. Banerjeea, V. Volpertbcd, Spatio-temporal pattern formation in RosenzweigCMacArthur model: Effect of nonlocal interactions, Ecological Complexity, 30(2017) $2-10$.

[18] M. Moustafa, M. H.Mohd, A. I. Ismail, F, A. Abdullah, Dynamical analysis of a fractional-order RosenzweigCMacArthur model incorporating a prey refuge, Chaos, Solitons, Fractals,109,(2018) 1-13.

[19] J. Sugie, Y. Saito, Uniqueness of limit cycles in a Rosenzweig-Macarthur model with prey immigration, SIAM J. Appl. Math., 72 (2012) 299-316.

[20] G. Zhu, J. J. Wei,Global stability and bifurcation analysis of a delayed predatorCprey system with prey immigration, Elec. J. Qual. Theo. Diff. Eqn., (2016).

[21] J. Hale, Theory of functional differential equation, Springer-Verlag, New York, 1977.

[22] S. Ruan, J. Wei, On the zeros of transcentental functions with applications to stability if delay differential equations with two delays. Dyn. Contin. Discrete Impus. Syst. Ser. A Math. Anal., 10 (2003) 863-874.

[23] S. Wiggins, Introduction to applied nonlinear dynamical systems and chaos, Springer, New York, 1990.

[24] T. Faria, L. Magalhães, Normal forms for retarded functional differential equations and applications to Bagdanov-Takens singularity, J. Differential Equations, 122 (1995) 201-224. 
[25] T. Faria, L. Magalhães, Normal forms for retarded functional differential equations with parameters and applications to Hopf bifurcation, J. Differential Equations, 122 (1995) 181-200.

[26] J. Wu, Symmetric functional differential equations and neural networks with memory, Trans. Am. Math. Soc., 350 (1998) 4799-838.

[27] B. Hassard, N. Kazarinoff, Y. Wan, Theory and Applications of Hopf Bifurcation, Cambridge Univ. Press, Cambridge, 1981. 